\title{
BACKGROUND DIFFERENCING ALGORITHM FOR MOVING OBJECT DETECTION USING SYSTEM GENERATOR HW/SW CO-SIMULATION
}

\author{
Dongre Sachinkumar ${ }^{1}$, Lakshmipriya $\mathrm{W}^{2}$ \\ ${ }^{1}$ Student, Department of E \& CE(VLSI \& ES), TJIT Bangalore, Karnataka, India \\ ${ }^{2}$ Assistant Professor, Department of E \& CE, TJIT Bangalore, Karnataka, India
}

\begin{abstract}
Background differencing algorithm was developed to detect moving objects from a stable system in which visual surveillance plays a major role. Among all existing algorithms it was chosen because of low computational complexity which is the major parameter of time in VLSI. The concept of the background differencing is to subtract the current image with respect to the reference image and compare it with to the certain threshold values. To check the background difference we have written the core processor Micro blaze is designed in VHDL (VHSIC hardware description language), implemented using SYSTEM GENERATOR. The test results are checked. The area and the speed are evaluated.
\end{abstract}

Keywords: Background Subtraction, Micro blaze, Object Detection, UART, VHDL.

\section{INTRODUCTION}

Object detection and tracking are important initial step in object recognition, tasks in computer vision. Object detection and tracking help us in video surveillance, it assists understanding the movement patterns of people to uncover suspicious events. It is also help full in traffic management to estimate flux and congestion statistics. In order to avoid the collision. Advanced vehicle control systems depend on the tracking information to keep the vehicle in lane and prevent from collisions. By using the object detection and tracking we can able to architecture design in retail space instrumentation by learning the shopping behavior of customers by tracking. In robotics also it play a key role[3]. Tracking is also a initial step or we can say a basic technology to extract regions of interest and video object layers as defined in different image formats(i.e. JPEG-2000 and MPEG-4 standards). In real-life systems also helpfull, and are required to track objects not only when the background of image is constant but also when lighting changes suddenly, motion of camera becomes large, also color contrast becomes low, image noise soars to an unacceptable level, etc. In addition, if we are considering the real time conditions than the computational complexity must keep minimum.

\section{REVIEW OF PREVIOUS ALGORITHMS}

There are so many approaches for moving detection object, here considering the optical flow, the temporal difference and background differencing.

\subsection{Optical Flow Method}

In a video frame, the field of motion vector per pixel or subpixel is called as flow of optical. There are so many methods by using those we can compute optical flow among which few are partial differential equation based methods, gradient consistency based methods and least squared methods[6]. The objective in optic flow calculation is to find the two-dimensional motion in an image sequence. As a pixel at location $(x, y, t)$ with intensity $I(x, y, t)$, than will have moved by $\delta x, \delta y$ and $\delta t$ between the two frames the equation can be given:

$$
I(x, y, t)=I(x+\delta x, y+\delta y, t+\delta t)
$$

Assuming that the movement is small enough, the above equation can be derived by using Taylor series as well. The main disadvantage of this optical flow is that due to high computational cost,hard to a apply in real-time.

\subsection{Temporal Difference Method}

The Frame difference is a part of background difference. In frame difference the current frame is simply subtracted from the previous frame, and the pixel values are different for different frames.

A major drawback of this method is the interior pixels, these interior pixels are interpreted as part of background. Another flaw is that the objects must move continuously [7]. This temporal differencing method is very adjustable to dynamic environments, but this method fails to extract all relevant pixels. Therefore we are using background differencing algorithm instead of these methods. 


\section{PROPOSED BACKGROUND MODELING}

Background differencing is a technique which is commonly used for segmenting out objects of interest in a image for different applications. This method compares an observed image with an estimate image if it contained no objects of interest. The name "background differencing" comes from the simple technique of subtracting the captured image from the estimated image and thresholding the result to generate the objects of interest.

Here we are checking several techniques and compare three important topics, how the object areas are different from the background, how the background is maintained over time and how the segmented object areas are post-processed to reject false positives, etc. In order to allow high-resolution images of the people in the scene to be acquired it is reasonable to assume that such people move about in the scene. To monitor the scene reliably it is essential that the processing time per frame be as low as possible. Hence it is important that the techniques which are employed are as simple and as efficient as possible. For that reason the well known technique of background differencing $[7,8]$ was selected for this application. Background differencing allows moving objects to be detected by taking the point-by-point absolute difference of the current image and a background image which must be acquired when there are no moving objects in the scene

$$
\text { Movingt }(i, j)=|\operatorname{Imaget}(i, j)-\operatorname{Background}(i, j)|
$$

When the subtracted value is more than the threshold value it is considered as a foreground otherwise it is background object.

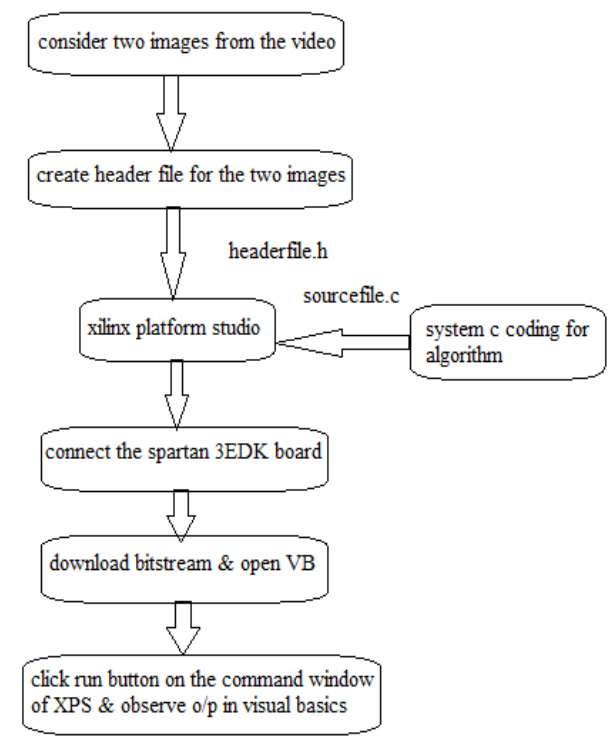

Fig -1: Flow diagram for proposed algorithm
In the first step we consider the video and convert it into frames, from that frames we can select any of two images for that two images we create a header file.And then we can write an algorithm for back ground subtraction in the XPS tool by using system ' $C$ ' language. In the XPS we are giving two inputs of headerfile.h and source file.c. XPS is a tool used to link a program to hardware like FPGA. After downloading the bit stream open the visual basics application and click run button on the command window of XPS .Finally with the help of UART port and RS232 cable we made a communication between hard ware and our PC and we can see the output image in the PC with the help of visual basics tool.

\subsection{Xilinx Platform Studio}

It is the development environment or GUI used for designing the hardware portion of embedded processor system. Xilinx embedded development kit is an integrated software tool suite for developing embedded systems with xilinx microblaze and CPUs. System design consists of the creation of the hardware and software components of the embedded processor system and the creation of a verification component is optional. Base System is the wizard that is used to automatically generate a hardware platform according to the user specifications that is defmed by the microprocessor hardware specification file. The microprocessor hardware specification file defines the system architecture, peripherals and embedded processors. The platform generation tool creates the hardware platform using the microprocessor hardware specification file as input. The software platform is defined by MSS (Microprocessor Software Specification) file which defines customizing operating systems (OS), libraries, and drivers and the The User Constraints File (UCF) specifies timing and placement constraints for the FPGA Design

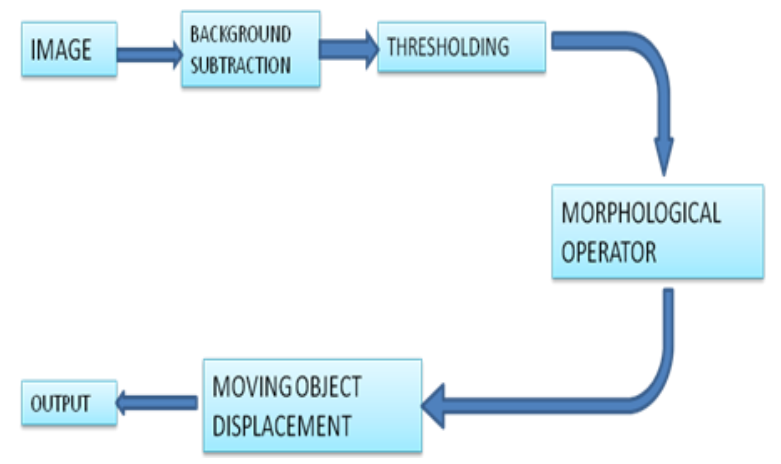

Fig -2: Block diagram

\subsection{Downloading Bitstream to FPGA}

When you select device configuration in that download bitstream, XPS downloads the bitstream (download.bit file) onto the target board using impact in batch mode. XPS uses the file etc/download.cmd for downloading the bit stream. 

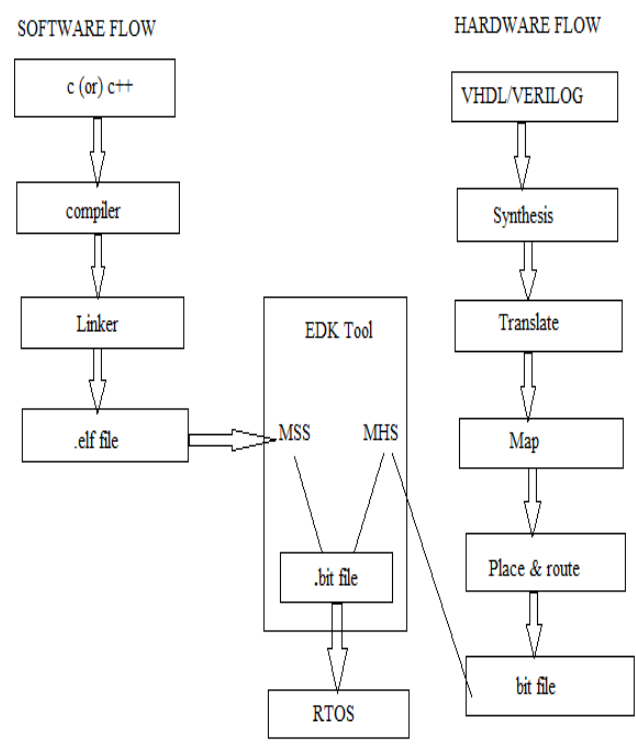

Fig -3: Embedded Development Kit Design Flow

The VB programming system packages up the complexity of windows in a truly amazing way. It provides simplicity and ease of use without sacrificing performance or the graphical features that make window such a pleasant environment to work in Menus, fonts, dialog, boxes etc are easily designed and these features require no more than a few lines of programming to control.

\subsection{Downloading Bitstream to FPGA}

When you select device configuration in that download bitstream, XPS downloads the bitstream (download.bit file) onto the target board using impact in batch mode. XPS uses the file etc/download.cmd for downloading the bit stream.

\section{EXPERIMENTAL RESULTS}

These are the object detection results in the visual basics tool the first two are the input images one with the object and the one with only background. Finally in the output image we can observe the object without background.

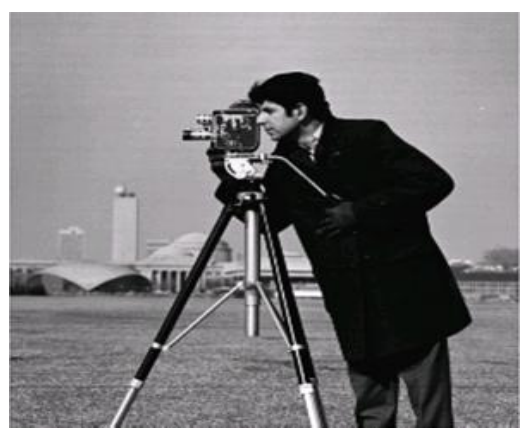

Fig -4: image display

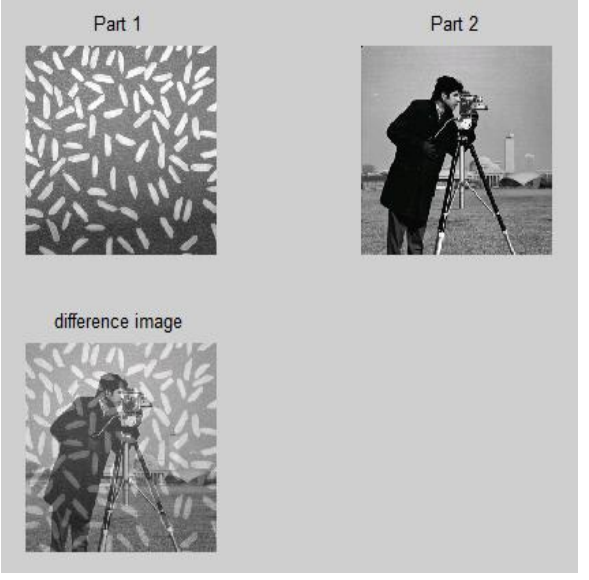

Fig -5: difference image

Table -1: Device Utilization Summary

\begin{tabular}{|l|c|c|c|}
\hline \multicolumn{1}{|l|}{ Logic utilization } & Used & Available & Utilization \\
\hline & & 1920 & $97 \%$ \\
Number of slices & 1880 & 3840 & $55 \%$ \\
Number of slice Flip & 2118 & & \\
flops & & 3840 & $77 \%$ \\
Number of 4i/p LUTS & 2971 & & \\
Number of Bonded & & 97 & $63 \%$ \\
IOBS & 62 & 12 & $33 \%$ \\
Number of BRAMS & 4 & 12 & $25 \%$ \\
Number of MUXES & 3 & 8 & $50 \%$ \\
Number of DCM's & 4 & 4 & $25 \%$ \\
Number of BSCAN's & 1 & & \\
\hline
\end{tabular}

\section{CONCLUSIONS}

Moving object detection based on background differencing algorithm designed on a reconfigurable hardware. When compared to the other algorithms like temporal difference and optical flow methods our background subtraction gives a better performance in the utilization of memory and logic elements.

\section{REFERENCES}

[1]. J. L. Barron, D. J. Fleet, and S. S. Beauchemin, "Performance of optical flow techniques," Int. J. Comput. Vis., vol. 12, no. 1, pp. 42-77, 1994

[2]. L. Maddalena and A. Petrosino, "A self-organizing approach to detection of moving patterns for real-time applications," in Proc. 2nd Int. Symp. Brain, Vision, and Artificial Intelligence, 2007, pp. 181-190, Lecture Notes Comput. Sci. 4729 
[3]. G. Backer, B. Mertsching, and M. Bollmann, "Data- and model-driven gaze control for an active-vision system," IEEE Trans. Pattern Anal

[4]. G.L.Foresti,"A Real Time System for Video Surveillance of Unattended Outdoor Environments".

[5]. K. Kim, T. H. Chalidabhongse, D. Harwood, and L. S. Davis, "Real-time foreground-background segmentation using codebook Model," Real-Time Imag., vol. 11, pp. 172-185, 2005.

[6]. A. Elgammal, D. Hanvood, and L. S. Davis, "Nonparametric model for background subtraction," in Proc. ECCV, 2000, pp. 751-767

[7]. C. Wren, A. Azarbayejani, T. Darrell, and A. Pentland, "Pfinder: Realtime tracking of the human body," IEEE Trans. Pattern Anal. Mach. Intell., vol. 19, no. 7, pp. 780-785, May1997.

[8]. R. J. Radke, S. Andra, O. Al-Kofahi, and B. Roysam, "Image change detection algorithms: a systematicsurvey,'IEEE Trans. Image Process., vol. 14, no. 3, pp. 294-307, Mar. 2005.

[9]. S.-C. Cheung and C. Kamath, "Robust techniques for background subtraction in urban traffic video," in Proc. EIVCIP, 2004, pp. 881-892.

[10]. M. Piccardi, "Background subtraction techniques areview," in Proc.IEEE Int. Conf. Systems, Man, Cybernetics, 2004, pp. 3099-3104.

[11]. R. T. Collins, A. J. Lipton, T. Kanade, H. Fujiyoshi, D. Duggins, Y. Tsin, D. Tolliver, N. Enomoto, O. Hasegawa, P. Burt, and L. Wixson, "A system for video surveillance and monitoring," Tech. Rep. CMU-RI-TR-00-12, The Robotics Inst., Carnegie Mellon Univ., Pittsburgh, PA, 2000.

[12]. K. Toyama, J. Krumm, B. Brumitt, and B. Meyers, "Wallflower: principles and practice of background maintenance," in Proc. 7th IEEE Conf. Computer Vision, 1999, vol. 1, pp. 255-261.

[13]. R. T. Collins, A. J. Lipton, T. Kanade, H. Fujiyoshi, D. Duggins, Y. Tsin, D. Tolliver, N.\ Enomoto, O. Hasegawa, P. Burt, and L. Wixson, "A system for video surveillance and monitoring," Tech. Rep. CMU-RI-TR-00-12, The Robotics Inst., Carnegie Mellon Univ., Pittsburgh, PA, 2000.

[14]. http://www.xilinx.com/training/xilinx-training courses.pdf

[15]. http://www.xilinx.com/xilinx10/books/docs/qst/qst.pdf

[16]. http://en.wikipedia.org/wiki/VHDL

[17]. http://www.vhdl-online.de/tutorial/

[18]. G.L.Foresti,"A Real Time System for Video Surveillance of Unattended Outdoor Environments".

[19]. G. Backer, B. Mertsching, and M. Bollmann, "Data- and model-driven gaze control for an active-vision system," IEEE Trans. Pattern Anal

\section{REFERENCES}

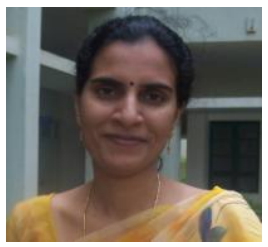

Asst. Prof. Lakshmipriya W, As an Assistant Professor working in TJIT, ME communication systems from Anna University.

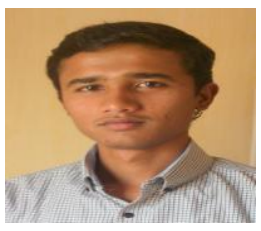

Mr. Dongre Sachinkumar, student of Mtech IV sem of Visvesvaraya Technological University Karnataka, I have completed my B.E from BKIT, Bidar in 2012 in Electronics \& Communication Engineering under the university of V. T. U Karnataka. 